\title{
Phase Transitions of Confined Lattice Homopolymers ${ }^{\dagger}$
}

\author{
Owen J. Hehmeyer, Gaurav Arya, and A. Z. Panagiotopoulos* \\ Department of Chemical Engineering, Princeton University, Princeton, New Jersey 08544
}

Received: November 25, 2003; In Final Form: February 9, 2004

\begin{abstract}
The effect of confinement on the phase behavior of lattice homopolymers has been studied using grand canonical Monte Carlo simulations in conjunction with multihistogram reweighting. The scaling of critical parameters and chain dimensions with chain length was determined for lattice homopolymers of up to 1024 beads in strictly $2 \mathrm{D}$ and quasi-2D (slab) geometries. The inverse critical temperature scales linearly with the ShultzFlory parameter for quasi-2D geometries, as it does for the bulk system. The critical volume fraction scales as a power law for all systems, with exponents $0.110 \pm 0.024$ and $0.129 \pm 0.004$ for the strictly 2D and slab geometries, respectively. The influence of confinement on critical behavior persists even in a thick slab due to the diverging correlation length of density fluctuations. The scaling of the radius of gyration with chain length in the quasi-2D system increasingly resembles the scaling in the strictly $2 \mathrm{D}$ system as the chain length increases. At the extrapolated infinite chain critical temperature, the radius of gyration of the 2D system scales with chain length with exponent $0.56 \pm 0.01 \simeq(4 / 7)$, in agreement with theoretical predictions.
\end{abstract}

\section{Introduction}

Polymer behavior in confined geometries and on surfaces is often of interest in biological, thin film, and semiconductor processes. The structure of polymers near surfaces is relevant to protein adsorption. ${ }^{1}$ Much recent effort has concentrated on understanding bulk polymer behavior, but atomistically detailed systems are often unable to capture the phase behavior of polymer solutions. Lattice models of polymers are popular because they can often describe phase behavior and critical phenomena without requiring the computational resources of simulations of complex atomistic models.

Recent studies of the phase behavior of lattice homopolymers $^{2-5}$ focus on the scaling of critical temperature and critical volume with chain length. The critical temperature, $T_{\mathrm{c}}$, generally increases with increasing chain length, $r$, according to the Shultz-Flory relationship ${ }^{6}$

$$
\frac{1}{T_{\mathrm{c}}(r)}-\frac{1}{T_{\mathrm{c}}(\infty)} \propto \frac{1}{\sqrt{r}}+\frac{1}{2 r}
$$

The critical volume fraction, $\phi_{\mathrm{c}}$, scales as a power law

$$
\phi_{\mathrm{c}}(r) \propto r^{-x_{2}}
$$

Many previous studies $2,3,7,8$ on the bulk behavior of lattice homopolymers have confirmed that Flory-Huggins theory can be used to describe the scaling of critical temperature with chain length for bulk three-dimensional (3D) systems. It was found in ref 3 that the critical volume fraction does not scale exactly as a power law but has some degree of curvature. Nonetheless, a power law fit gives $x_{2}=0.39 \pm 0.02$, which is in good agreement with experiment ${ }^{9}$ and other simulations ${ }^{2,4,8}$ over a comparable range of chain lengths. Yan and dePablo ${ }^{4}$ more recently studied the same simple cubic lattice polymer system but used a newly developed expanded ensemble grand canonical

* Corresponding author. E-mail: azp@princeton.edu.

$\dagger$ Part of the special issue "Hans C. Andersen Festschrift". simulation that enabled them to study chains up to lengths of $r$ $=16000$. They observed curvature in the critical volume vs chain length plot that suggests that the critical volume, in the limit of infinite chain length, scales with exponent $x_{2} \simeq 0.5$, as Flory suggested. ${ }^{10}$ Yelash et al. ${ }^{11}$ recently completed an exhaustive review of the experimental, theoretical, and simulation results for the scaling of the critical volume with chain length and proposed a new correlation.

Two-dimensional lattice homopolymers are less extensively studied than polymers on a 3D lattice. The structure of strictly two-dimensional (2D) athermal polymers was recently investigated by Yethiraj, ${ }^{12}$ who studied the radius of gyration, $R_{\mathrm{g}}$, over a range of densities, confirming that $R_{\mathrm{g}} \sim r^{0.75}$ in the limit of low density, in agreement with the theoretical work of Nienhuis ${ }^{13}$ and Flory and Huggins excluded volume theory, which predicts

$$
v=3 /(d+2)
$$

where $d$ is the number of dimensions. Flory's excluded volume theory, though mean-field in character, gives the exact exponent for the 2D case, and overestimates only slightly for the 3D case. Yethiraj also shows that the scaling parameter decreases as density increases and reviews other studies ${ }^{14-16}$ of lattice polymers in 2D systems that have focused on the scaling of the radius of gyration. Dickman ${ }^{14}$ and Reiter ${ }^{16}$ have simulated 2D chains at the $\Theta$ temperature and compared results to theory on self-avoiding walks, ${ }^{17}$ which suggest that at the $\Theta$ temperature, $R_{\mathrm{g}} \sim r^{4 / 7} \simeq 0.5714$. Much more is known about the radius of gyration (structure) in 2D than about the critical behavior (thermodynamics), which Binder et al..$^{5}$ have studied recently. They examined the configurations of strictly 2D polymer blends using the bond fluctuation model. They find that $T_{\mathrm{c}} \sim \sqrt{N}$ rather than $T_{\mathrm{c}} \sim N$, the bulk relationship. They conclude that the strong screening of interchain interactions that occurs in a 2D geometry causes this effect. They also predict that such behavior might be observed in films where the thickness is comparable to the excluded volume correlation length. 
Studies of confined polymers in a geometry periodic in $x$ and $y$, but containing some finite dimension $z$, sometimes called a slit, slab, or quasi-2D geometry are even less frequent. Bleha and $\mathrm{Cifra}^{18}$ have studied the free energy and force experienced by a polymer chain confined in a slit in equilibrium with a bulk solution. They also examined the density profile across the $z$ direction of the quasi-2D geometry. Teraoka and Wang ${ }^{19}$ studied polymers in a slab geometry, focusing on the shape of polymers in various slab widths.

The present study focuses on the thermodynamics of confined lattice homopolymer systems. These can be thought of as a onecomponent polymeric system in an incompressible solvent of varying quality confined in narrow slits. The critical parameters are obtained through mixed-field finite-size scaling methods as functions of chain length and gap width. In contrast to many prior studies, the structure (radius of gyration) is examined in relationship to the thermodynamics. This connection is made through two definitions of the $\Theta$ temperature, which is found by extrapolation to the infinite chain length critical temperature and by examination of the scaling of $R_{\mathrm{g}}$. The overarching aim of this study is to delineate more clearly the difference between strictly 2D, "nearly" 2D, and 3D systems.

The paper is organized as follows. First, the computer simulation methods used in this work are introduced. Next, the scaling of critical properties with chain length is discussed. The scaling of the radius of gyration with chain length is presented next. The paper ends with conclusions regarding the qualitative effects of confinement on structure and thermodynamics.

\section{Model and Simulation Methods}

2.1. Grand Canonical Monte Carlo. All simulations were carried out in the grand canonical ensemble, at fixed chemical potential, $\mu$, volume, $V$, and temperature, $T$. Chain lengths of powers of 2 were studied, from $r=1$ to $r=1024$. Polymer chains were generated with the configurational bias method of Rosenbluth and Rosenbluth. ${ }^{20}$ Microstates were generated with creation or annihilation, reptation, and cluster moves. Chains of $r=1$ were simulated with only creation and annihilation moves, and chains of $r=2$ with $99.9 \%$ creation and annihilation and $0.1 \%$ cluster moves. Chains of $4 \leq r \leq 128$ were simulated with a mix of moves that was $70 \%$ creation or annihilation, $29.9 \%$ reptation, and $0.1 \%$ cluster moves; ${ }^{21}$ For $r=256$ we used a $60 \% / 39.9 \% / 0.1 \%$ mix. Chains of $r=512$ and $r=1024$ were simulated with the expanded ensemble method and are discussed in section 2.2. Moves were accepted or rejected according to Metropolis criteria. ${ }^{21}$ Acceptance rates for the creation/annihilation moves for the slab geometry and near the critical point were roughly inversely proportional to chain length and fell from $30 \%$ to $3 \%$ at the longest chain length of $r=256$ studied using "standard" grand canonical Monte Carlo (GCMC). Acceptance rates for the strictly 2D geometry were lower, falling to $1.5 \%$ at $r=256$. The number of Monte Carlo (MC) steps after equilibrium in the quasi-2D system ranged between $2 \times$ $10^{8}$ and $2.5 \times 10^{9}$, depending on chain length. Because of lower acceptance, the number of MC steps used in strictly 2D systems ranged from $5 \times 10^{8}$ to $4 \times 10^{9}$. The longest simulations of the $r=256$ chains took 3 days on Pentium 4 processors running at $2 \mathrm{GHz}$.

2.2. Expanded Grand Canonical Monte Carlo. For polymer chains with $r=512$ and 1024 beads, the standard GCMC simulation method becomes inefficient due to increased difficulties in inserting and deleting these long chains. To increase efficiency, we adopted the expanded ensemble formalism of Escobedo and de Pablo ${ }^{22}$ for insertions and deletions. The basic idea of this approach is to maintain a system composed of a number of full chains plus a single tagged chain of an intermediate length. The Monte Carlo steps then involve inserting/deleting polymer segments of size $\Delta$ beads to/from the tagged chain at a time, as opposed to the entire chain in the regular GCMC simulations. The different states corresponding to varying lengths of the tagged molecule form the expanded states of the expanded GCMC partition function (a total number of $r / \Delta$ states). The corresponding preweighting factors associated with different states were chosen to be equal to the incremental chemical potential $\mu^{\prime}=\mu \Delta / r$, where $\mu$ is the chemical potential corresponding to a full chain insertion or deletion. This was done so as to maintain a uniform frequency of sampling between the various states. ${ }^{23}$ For our systems, we find that $\Delta=64$ beads result in the most efficient simulations for both the $r=512$ and 1024 chains near their respective critical points. For simulations of $r=1024$ in the quasi-2D geometry, acceptance rates increased by a factor of 6 over the rates observed in standard GCMC simulations. However, in the strictly 2D geometry, we obtain computational savings of factors of 10 and 400 for chains of $r=512$ and $r=1024$, respectively.

2.3. Determination of Critical Parameters. The critical point is the state point where density fluctuations become infinite at the thermodynamic limit and the phase boundary disappears. This unique point characterizes the thermodynamics of a system and the scaling of critical properties with chain length at that point is of primary interest for this study. Through cycles of conducting a series of simulations, histogram reweighting, and then narrowing in on the critical point, as explained later in this section, the critical temperature and density for each chain length were found.

For both standard and expanded GCMC simulations, after equilibrium was reached, histograms were collected. Each histogram records the number of times the simulation visits a state with $N$ particles and an energy within a small range. Multihistogram reweighting was used to combine the histograms according to the method of Ferrenberg and Swendsen. ${ }^{24,25}$ Near the critical point, the simulation visits many different states because of large density fluctuations. The combined histograms provide a self-consistent estimate of the probability distribution function $P_{\mu, \beta}(N, E)$ which can be used to extrapolate to a new state point.

The finite-size scaling concepts of Wilding et al. ${ }^{26,27}$ were used for locating the critical points of both strictly $2 \mathrm{D}$ and quasi2D systems. Finite-size scaling theory defines an ordering operator, $M$, as a combination of the number of particles, $N$, and the energy, $E, M=N-s E$, where $s$ is a field mixing parameter that controls the strength of the coupling between $N$ and the fluctuations in $E$ near the critical point. At the critical point the probability distribution for a given system, $P_{\mathrm{L}}(x)$, where the scaling parameter is given by $x=a(L, r)\left(M-M_{\mathrm{c}}\right)$, assumes the universal shape corresponding to Ising-type criticality for short-range interactions. The nonuniversal parameter $a(L, \boldsymbol{r})$, where $r$ is a system-specific quantity controlling the degree of field mixing, is chosen so that unit variance is observed in the scaling parameter. The distribution is reweighted to find the chemical potential and temperature that result in the best match of the observed data and the universal $P_{\mathrm{L}}(x)$ for the twodimensional Ising distribution. Data for the quasi-2D system were also matched to the two-dimensional Ising distribution. Attempts at matching the 3D distribution were also made, but the shape of the distribution for the quasi-2D data did not match with the 3D distribution. An example of matching data for $r=$ 64 to the $2 \mathrm{D}$ universal curve is shown in Figure 1. 


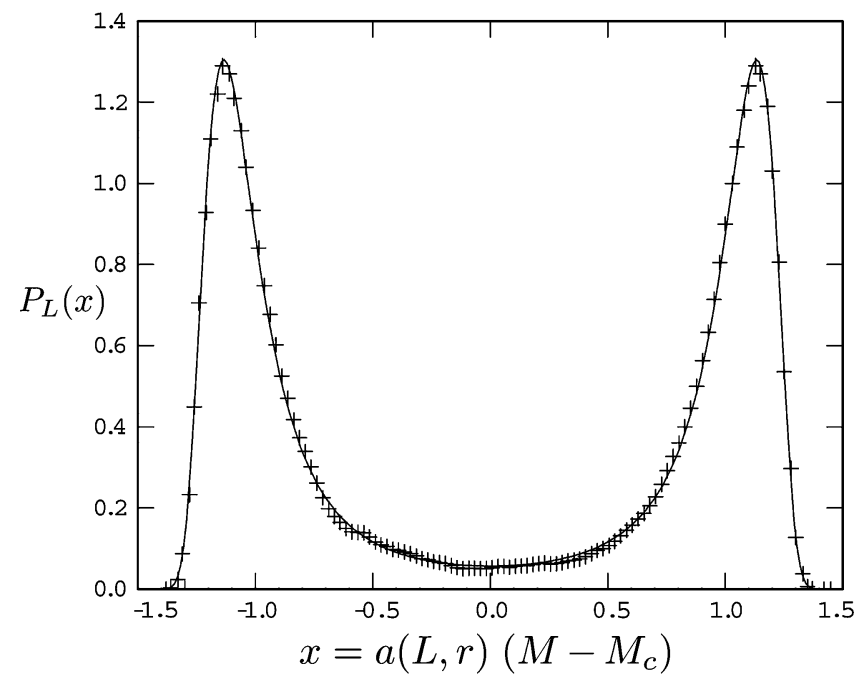

Figure 1. Matching of the probability distribution, $P_{\mathrm{L}}(x)$, where the scaling parameter is given by $x=a(L, r)\left(M-M_{\mathrm{c}}\right)$ to the universal 2D Ising curve, indicated by the continuous line. Data points $(+)$ are for chains of length $r=64$ in a slab of width $h=3$.

2.4. Model System. Homopolymers of length $r$ were studied on a cubic lattice of coordination number $Z=26$. The possible relative position vectors between successive beads are $(0,0,1)$, $(0,1,1),(1,1,1)$, and their equivalents resulting from symmetry operations. Polymer beads interact using nearest neighbor pairwise interactions with an energy parameter of $\epsilon=-1$. Interactions with the solvent are defined to be zero. Reduced temperature is defined as

$$
T^{*}=\frac{k T}{|\epsilon|}
$$

where $k$ is Boltzmann's constant. The GCMC simulations were carried out in strictly 2D and slab geometries. In all cases, periodic boundary conditions were applied in the $x$ and $y$ directions. To study the effect of confinement, we have constrained the simulation box between two impenetrable walls in the $z$ direction, creating a slab geometry. This geometry, finite in the $z$ direction, will be referred to as the "quasi-2D" or slab geometry. The thickness of a slab is characterized in this study by the number of lattice spaces in the $z$ direction, $h$. The length in the $x$ and $y$ directions is $L$ lattice spacings. Reduced density is defined as

$$
\phi=\frac{r N}{V}
$$

where the volume is given by $V=L^{2} h, r$ is the number of beads, and $N$ the number of polymers molecules. The strictly 2D geometry is the special case of the quasi-2D geometry where $h$ $=1$ lattice spacing. Schematic depictions of two typical configurations of a polymer of $r=16$ in strictly 2D and quasi2D geometries are shown in Figure 2. The slab geometry $h=$ 3 was studied extensively because its phase behavior was sufficiently different from the $2 \mathrm{D}$ and $3 \mathrm{D}$ geometries to be of interest.

\section{Results and Discussion}

3.1. Scaling of Critical Parameters. The apparent critical parameters of the 2D and quasi-2D systems studied herein are listed in Table 1. Critical data for 3D systems has been previously reported in ref 3 . Throughout this work, critical data for the bulk (3D) systems are included in figures for facilitation

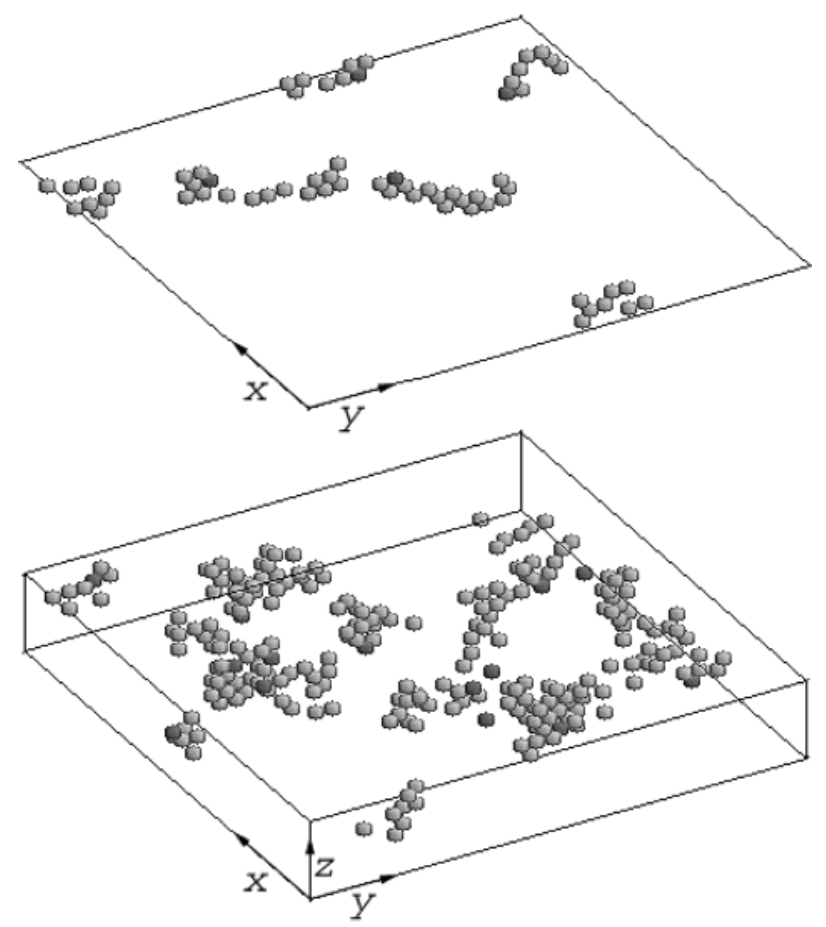

Figure 2. Typical configurations of a strictly $2 \mathrm{D}$ system $(h=1)$ and a "quasi-2D" system $(h=3)$. The first bead of each polymer is shaded differently to aid identification of each polymer.

TABLE 1: Critical Temperature, $T_{\mathrm{c}}^{*}$, Density, $\phi_{\mathrm{c}}$, and Chemical Potential, $\mu_{c}$, as a Function of Chain Length, $r$, and System Size, $L$, for Strictly 2D and Quasi-2D Geometries $^{a}$

\begin{tabular}{rrrccc}
\hline \multicolumn{1}{c}{$h$} & \multicolumn{1}{c}{$L$} & \multicolumn{1}{c}{$T_{\mathrm{c}}^{*}$} & $\phi_{\mathrm{c}}$ & $\mu_{\mathrm{c}}$ \\
\hline 1 & 1 & 32 & $1.314(3)$ & $0.500(3)$ & $-4.000(2)$ \\
& 2 & 32 & $1.655(3)$ & $0.468(1)$ & $-4.805(4)$ \\
& 4 & 64 & $1.972(2)$ & $0.445(2)$ & $-3.532(8)$ \\
& 8 & 64 & $2.269(3)$ & $0.423(1)$ & $3.31(3)$ \\
& 16 & 64 & $2.533(1)$ & $0.393(2)$ & $23.06(3)$ \\
& 16 & 128 & $2.533(2)$ & $0.394(1)$ & $23.06(4)$ \\
& 32 & 128 & $2.758(1)$ & $0.368(1)$ & $71.10(3)$ \\
& 64 & 256 & $2.942(2)$ & $0.341(5)$ & $178.7(1)$ \\
& 128 & 128 & $3.088(3)$ & $0.316(7)$ & $409.4(7)$ \\
& 256 & 256 & $3.197(7)$ & $0.291(1)$ & $891(3)$ \\
& 512 & 512 & $3.289(-)$ & $0.274(-)$ & $1891(-)$ \\
3 & 1024 & 512 & $3.358(-)$ & $0.250(-)$ & $3934(-)$ \\
& 1 & 32 & $3.998(2)$ & $0.462(1)$ & $-10.246(1)$ \\
& 2 & 32 & $5.196(8)$ & $0.406(3)$ & $-17.53(1)$ \\
& 4 & 48 & $6.40(2)$ & $0.365(4)$ & $-26.24(1)$ \\
& 8 & 64 & $7.522(6)$ & $0.327(3)$ & $-33.97(2)$ \\
& 16 & 64 & $8.498(3)$ & $0.295(1)$ & $-36.19(2)$ \\
& 16 & 96 & $8.503(4)$ & $0.295(2)$ & $-36.15(2)$ \\
& 32 & 96 & $9.325(5)$ & $0.267(2)$ & $-23.39(8)$ \\
& 64 & 128 & $9.99(2)$ & $0.243(5)$ & $23.5(8)$ \\
& 128 & 128 & $10.50(1)$ & $0.222(1)$ & $143.3(7)$ \\
& 256 & 256 & $10.90(2)$ & $0.203(1)$ & $415(4)$ \\
& 512 & 512 & $11.20(-)$ & $0.186(-)$ & $1004(-)$ \\
& 1024 & 512 & $11.40(-)$ & $0.169(-)$ & $2219(-)$ \\
& 16 & 64 & $10.452(2)$ & $0.245(1)$ & $-59.318(8)$ \\
& 16 & 64 & $12.137(3)$ & $0.202(1)$ & $-75.803(7)$
\end{tabular}

${ }^{a}$ The error in the last digit is shown in parentheses. Error was not calculated for those simulations completed using the expanded ensemble method, $r=512$ and $r=1024$.

of discussion, but only the radius of gyration calculations for the bulk were performed in the present study. From data away from the critical point, phase coexistence was determined by matching the area under the liquid and gas portions of the density distributions. For data very near the critical point, data were fit to scaling relationships as explained in section 2.3. The 


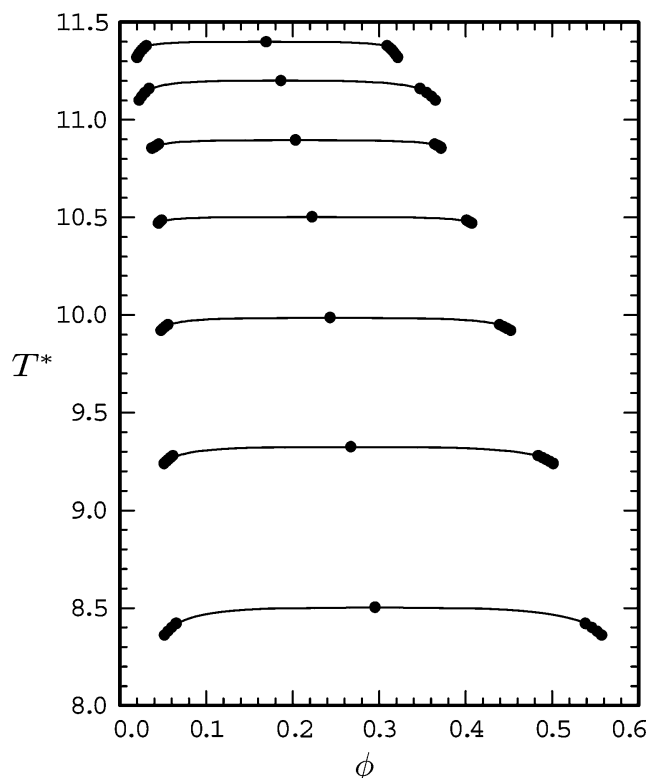

Figure 3. Reduced temperature, $T^{*}$, versus volume fraction for system with $h=3$. From bottom to top the curves are for $r=16,32,64,128$, 256,512 , and 1024, respectively. The circles denote those portions of the coexistence curve found through histogram reweighing. The center circle is the critical point. Lines are guides to the eye obtained from fitting the data to scaling relationships near the critical point.

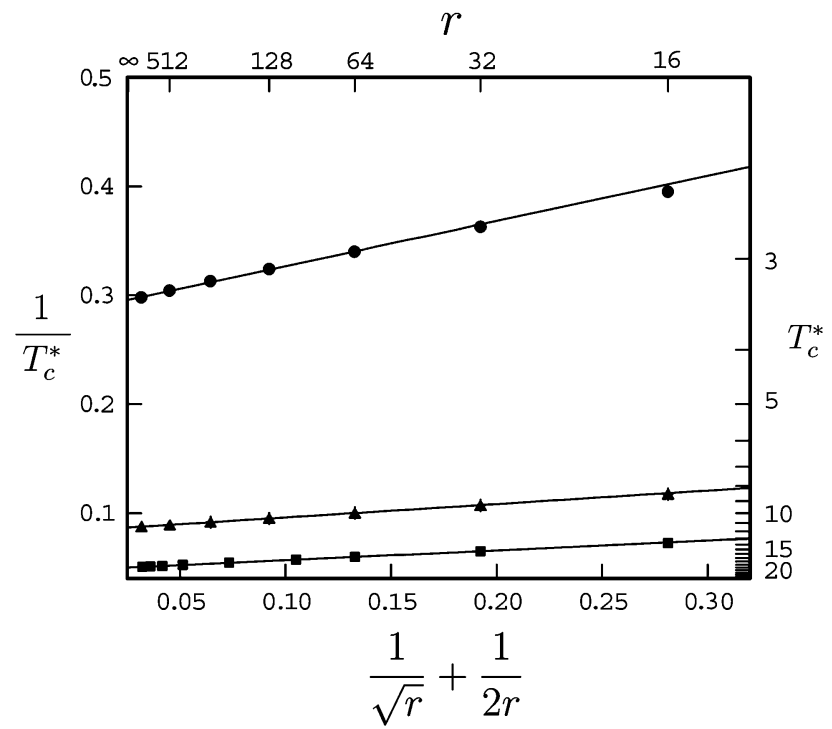

Figure 4. Inverse critical temperature, $1 / T_{\mathrm{c}}^{*}$, versus the Shultz-Flory parameter ( $r$ is chain length) for strictly $2 \mathrm{D}$ (circles), $h=3$ (triangles), and 3D geometries (squares). Lines are linear least-squares fit to data for $r \geq 64$. Statistical uncertainties are smaller than symbol size. Data for the 3D geometry are from Wong and Panagiotopoulos. ${ }^{3}$

temperature-density phase diagram for the quasi-2D systems with $h=3$ is shown in Figure 3.

Figure 4 shows a plot of inverse critical temperature vs the Shultz-Flory ${ }^{10}$ parameter for each of the three geometries. The relationship between $1 / T_{\mathrm{c}}^{*}$ and $(1 / \sqrt{r}+1 / 2 r)$ is increasingly linear as chain length increases. A linear least-squares fit was made using data for $r \geq 64$ for all three geometries. Fits give slopes of $0.41 \pm 0.04,0.123 \pm 0.001$, and $0.090 \pm 0.001$ for strictly 2D, slab, and 3D geometries, respectively. For the strictly 2D geometry (filled circles in Figure 4), the relationship between the Shultz-Flory parameter and $1 / T_{\mathrm{c}}^{*}$ is not linear but shows curvature even at the longest chain lengths studied. In the quasi2D and 3D geometries, the relationship is highly linear, with a correlation coefficient of $R^{2}>0.9998$. The critical temperature

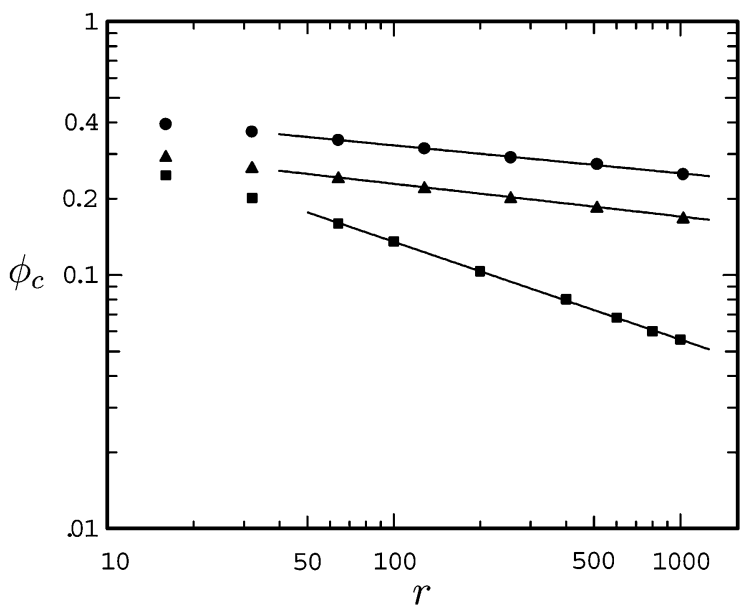

Figure 5. Critical volume fraction, $\phi_{\mathrm{c}}$, as a function of chain length, $r$, for strictly 2D (circles), slab of $h=3$ (triangles), and 3D geometries (squares). Statistical uncertainties are smaller than symbol size. Data for the 3D geometry are from Wong and Panagiotopoulos. ${ }^{3}$

behavior of the slab geometry appears more similar to the behavior of the $3 \mathrm{D}$ geometry than to the strictly $2 \mathrm{D}$ geometry, whose critical temperature rises more rapidly with the ShultzFlory parameter. Extrapolation to infinite chain length gives $T_{\mathrm{c}}^{*}(\infty)=11.94 \pm 0.01$ for the slab geometry. Similarly, for the strictly $2 \mathrm{D}$ system, $T_{\mathrm{c}}^{*}(\infty)=3.51 \pm 0.03$. The choice of the chain length cutoff does not affect the infinite chain length extrapolation. The estimated uncertainties in the infinite-chain length values were obtained from a combination of simulation statistical errors and the uncertainty over the linear range of extrapolation. In addition to the Shultz-Flory parameter, the $1 / T_{\mathrm{c}}^{*}$ data were also fit to the more general form, $1 / \sqrt{r}+A / r$. The extrapolated infinite chain length critical temperatures obtained from the fit are not statistically significantly different from the fit with the Shultz-Flory parameter with $r \geq 64$.

It is hypothesized ${ }^{3,7,28}$ that this extrapolation gives the $\Theta$ temperature, the temperature at which the attractive and repulsive forces between polymer segments exactly balance. The $\Theta$ temperature for bulk 3D systems is also defined to be the temperature where the second virial coefficient vanishes or the point where the polymer chain behaves as a random coil, meaning the mean end-to-end distance is directly proportional to the number of beads. Bruns ${ }^{28}$ demonstrated the equivalence of these later two definitions for a 3D system with coordination number $Z=6$. For this same 3D system, Panagiotopoulos and Wong $^{3}$ later showed that the extrapolation of the critical temperature to infinite chain length also provided the same estimate of the $\Theta$ temperature as these two earlier definitions. We discuss the $\Theta$ temperature in the context of confined systems in section 3.2.

The critical volume scales with chain length in a linear fashion when plotted on a $\log -\log$ scale, as shown in Figure 5. The slope of the 3D best fit line was previously established to be $x_{2}$ $=0.39 \pm 0.02 .{ }^{3}$ Fitting only data with $r \geq 64$, the slope of the strictly 2D curve gives $x_{2}=0.110 \pm 0.024$, and for the slab geometry, $x_{2}=0.129 \pm 0.004$. Unlike the critical temperature, the critical volume for the slab geometry scales more similarly to the strictly 2D geometry. At long chain lengths we expect these slopes to become the same, and they are already within the error bounds of one another. The slab geometry appears closer to 2D from the perspective of the scaling of the critical temperature, but closer to 3D from the standpoint of critical density. To answer the question of whether the slab geometry should rightfully be called a quasi-2D or a quasi-3D geometry, 


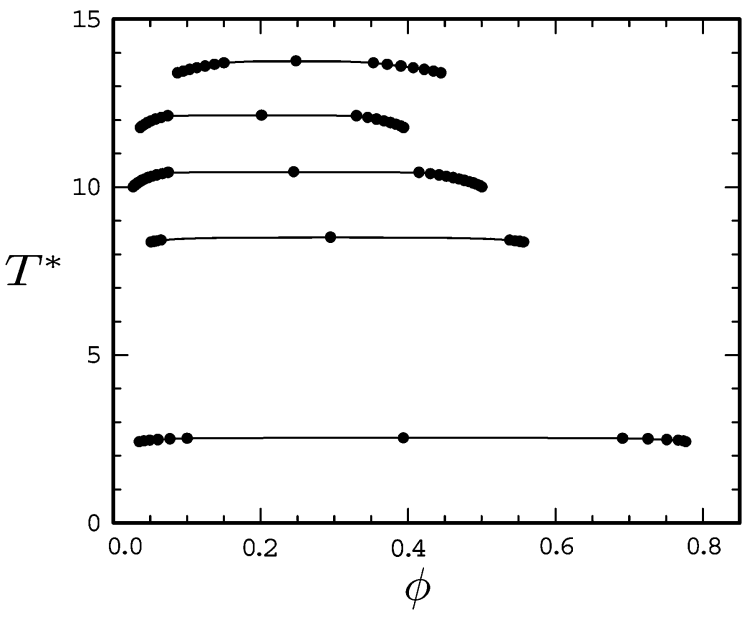

Figure 6. Reduced temperature, $T^{*}$, versus volume fraction for chains of length $r=16$. From bottom to top the curves are for strictly 2D, slabs of $h=3,5,10$, and 3D $(h \rightarrow \infty)$ geometries. Symbols and lines are as in Figure 3.

it is instructive to examine the phase diagram of a typical homopolymer.

Chains of length $r=16$ were studied in several different slab geometries of increasing thickness. In addition to systems of $h=1$ and $h=3$, simulations in systems of $h=5$ and $h=$ 10 were also completed. As in the studies of the $h=1$ and $h$ $=3$ geometries, the critical temperature and critical density were found, and a phase diagram in the temperature-density plane was constructed, shown here in Figure 6. As the thickness increases, the critical temperature and density increase, moving closer to the bulk value. However, even at $h=10$, roughly 4 times the radius of gyration, it is clear the critical temperature and density of the quasi-2D system do not reach the bulk value. This is because the density fluctuations at the critical point are of infinite correlation length. Thus, any finiteness in the $z$ direction will cause a large discrepancy between the slab geometry and the bulk geometry, whatever the thickness of the slab. The trend seen in the simulation data suggest that the location of the critical points of the confined systems will not converge smoothly to that of the $3 \mathrm{D}$ system as the wall separation is increased. Other works, including the work of Fischer and Nakanishi ${ }^{29}$ suggest otherwise. Interested readers should consult the review of Binder, Landau, and Müller ${ }^{30}$ for a comprehensive discussion of the effects of finiteness on criticality.

Another illustration of finiteness-induced effects is seen by examining the density profile of chains of length $r=16$ across the simulation box. Figure 7 shows density profiles for a range of densities, both above and below the critical density. The profiles were computed across a lattice of $h=30$ so effects at several multiples of the radius of gyration could be examined. Profiles were computed at $T^{*}=13.5$, near the estimated critical temperature. The critical density, $\phi_{\mathrm{c}}$, of a system of width $h=$ 30 is estimated to be about 0.15 from extrapolation of the studied systems. The profile is flat for low densities, rising and becoming more pointed near the critical density, and then falling again and becoming flat as the density increases further. The divergence of the boundary layer thickness at $\phi_{\mathrm{c}}$ is related to the divergence of the correlation length of the density fluctuations as $\phi \rightarrow \phi_{c}$. Away from the critical density, these fluctuations are finite and smaller than the width of the simulation box.

The critical points of the quasi-2D systems were found by matching the 2D Ising curve to the histogram data. Matching

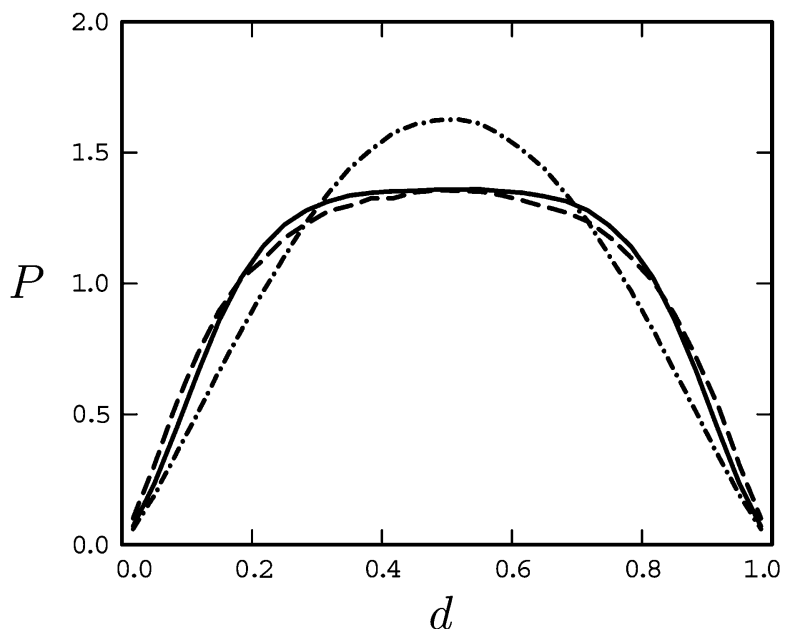

Figure 7. Density profile of chains of length $r=16$ in the $z$ direction across a lattice of $h=30$. The distance parameter, $d$, is the distance in lattice units from one wall normalized by the width of the lattice. The probability parameter, $P$, is the likelihood of finding a bead in the lattice space at $d$ normalized by the likelihood if all beads were distributed evenly. Profiles shown are for $\phi$ of 0.07 (dash), 0.16 (dot-dash), and 0.39 (solid), respectively, at a temperature of $T^{*}=13.5$.

to the $3 \mathrm{D}$ curve was also attempted, but the data did not fit the $3 \mathrm{D}$ Ising curve as well as it did the 2D curve. Nonetheless, minimizing the error between the data and the $3 \mathrm{D}$ curve as best as possible, we find that the apparent critical temperature increased by $1.2 \%$ and the critical density decreased by $1.9 \%$, which still does not put the critical parameters for quasi-2D systems on a trend toward the bulk value.

3.2. Scaling of the Radius of Gyration. The radius of gyration is strongly affected by temperature and, to a lesser degree, density. The radius of gyration was calculated for each length chain at a variety of low densities. Density was lowered until the radius of gyration showed no meaningful changes. The data shown were computed at very low densities, always much less than the crossover density from dilute to semidilute solutions:

$$
\phi^{*}=\frac{r}{R_{\mathrm{g}}^{\mathrm{d}}}
$$

The crossover density was computed and compared to $\phi$, and it was found that $\phi<\phi^{*}$ for the studied systems. Even at the longest chain lengths studied, $\phi$ was less than $1 \%$ for all geometries. Density tended to have a stronger effect on the longer chains because the crossover density decreases with increasing chain length. For this reason, $\phi / \phi^{*}$ was computed and was less than 0.1 for the longest chains studied.

Radii of gyration for selected systems at the athermal limit are shown in Figure 8 as a function of chain length. The data shown were computed at a temperature that was high enough to be effectively in the athermal limit in which only excluded volume interactions are relevant. A linear least-squares fit of the data in Figure 8 was made for chain lengths $r \geq 16$. The scaling exponents were found to be $v=0.58 \pm 0.02,0.69 \pm$ 0.03 , and $0.74 \pm 0.02$ for the $3 \mathrm{D}$, slab, and $2 \mathrm{D}$ geometries, respectively. The scaling of $R_{\mathrm{g}}$ for the $3 \mathrm{D}$ geometry is expected to be 0.59 from earlier work, ${ }^{31}$ which compares well with our value. Flory estimated the scaling exponent for the $3 \mathrm{D}$ case to be 0.6 using eq 3 , and applying it to the $2 \mathrm{D}$ case the scaling is estimated to be 0.75 . Our scaling of $0.74 \pm 0.02$ compares well with this value. Unlike the $2 \mathrm{D}$ and $3 \mathrm{D}$ cases, the value of the quasi-2D scaling exponent increases as the chain length cutoff 


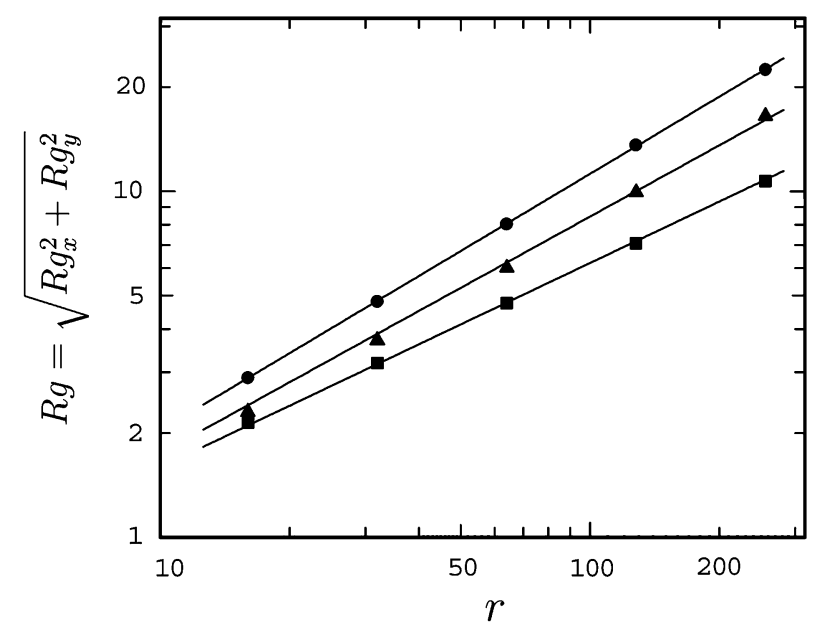

Figure 8. Scaling of $R_{\mathrm{g}}$ as a function of chain length for strictly 2D (circles), slab of $h=3$ (triangles), and 3D geometries (squares) for athermal systems.

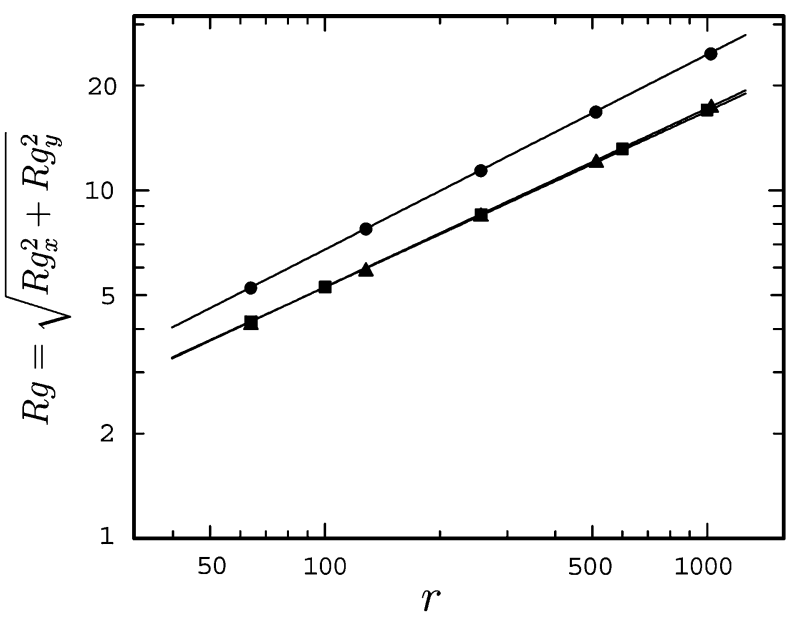

Figure 9. Scaling of $R_{\mathrm{g}}$ as a function of chain length for strictly 2D (circles), slab of $h=3$ (triangles), and 3D geometries (squares) for systems at $T_{\mathrm{c}}^{*}(r)=T_{\mathrm{c}}^{*}(\infty)$.

of the fit is increased, meaning the data are not linear. At longer chain lengths the true scaling exponent is higher, and trends toward the value of the $2 \mathrm{D}$ case because the polymer sees the geometry as increasingly $2 \mathrm{D}$ as chain length increases. For a small polymer, $r=8$, there is ample space in a geometry of $h$ $=3$ to coil into a roughly spherical shape because the width is greater than the radius of gyration. For a long chain polymer, significant extension takes place because the radius of gyration is much greater than the width of the slab.

The scaling of the radius of gyration at the extrapolated infinite chain length critical temperature, $T_{\mathrm{c}}^{*}(\infty)$, was found for strictly 2D, quasi-2D, and 3D systems of coordination number $Z=26$. Figure 9 shows the radii of gyration for these systems as a function of chain length. The radius of gyration scales as $R_{\mathrm{g}} \propto r^{0.505 \pm 0.006}$ at a temperature of $T_{\mathrm{c}}^{*}(\infty)=20.58$, the extrapolated $\Theta$ temperature of a 3D system with coordination number $Z=26$, as determined in previous study. ${ }^{3}$ This scaling is in agreement with the premise that the chain should behave as a random coil, $R_{\mathrm{g}} \propto r^{0.50}$, which lends further credence to the conjecture that these definitions really do provide the same estimate of the $\Theta$ temperature. For the 2D system, the scaling is $R_{\mathrm{g}} \propto r^{0.56 \pm 0.01}$ at $T_{\mathrm{c}}^{*}(\infty)=3.51$. This is consistent with the scaling theory of $R_{\mathrm{g}}$ at the $\Theta$ temperature, ${ }^{14,17}$ which predicts $R_{\mathrm{g}} \propto r^{4 / 7}$. Thus, for $2 \mathrm{D}$ systems, the extrapolated infinite chain length critical temperature corresponds to the $\Theta$ temperature.
In the quasi-2D system, the scaling is $R_{\mathrm{g}} \propto r^{0.52 \pm 0.01}$ at $T_{\mathrm{c}}^{*}(\infty)=$ 11.94 , but the exponent trends slightly higher with longer chain length cutoff. It is expected that for much longer chains than those studied here, the quasi-2D system's scaling will converge to that of the strictly $2 \mathrm{D}$ system.

Finally, a connection exists between $\phi_{\mathrm{c}}$ and the just discussed scaling of $R_{\mathrm{g}}$. By substituting $R_{\mathrm{g}} \sim r^{v}$ into eq 6 , the scaling of the radius of gyration can be related to the crossover density: ${ }^{31}$

$$
\phi^{*} \sim \frac{r}{R_{\mathrm{g}}^{\mathrm{d}}} \sim r^{1-d v}
$$

The critical density, $\phi_{\mathrm{c}}$, must scale as $x_{2} \leq d v-1$ because the crossover density would be larger than the critical density and the chains would not interact if $x_{2}$ were greater. For $d=3$ and $v=1 / 2, x_{2}=1 / 2$, the correct scaling within mean field theory. Applying the $2 \mathrm{D}$ simulation data from our study, $d v-1 \approx 2$ $\times 0.56-1=0.12$, which is very close to $x_{2} \approx 0.11$. Some radius of gyration calculations at $T^{*}=T_{\mathrm{c}}^{*}$ for each chain length were also performed, and these additional data are available from the authors.

\section{Conclusions}

We have investigated the phase behavior and structure of lattice homopolymers confined to a surface and between two hard walls using regular and expanded ensemble GCMC simulation. Chain lengths ranging from $r=1$ to $r=1024$ were investigated in a strictly 2D geometry $(h=1)$ and a slab geometry $h=3$ lattice units thick. Confinement alters critical behavior substantially from the behavior of polymers in bulk. The scaling of critical volume with chain length for a $2 \mathrm{D}$ geometry was found to be $x_{2}=0.110 \pm 0.024$, and for a quasi$2 \mathrm{D}$ geometry, $x_{2}=0.129 \pm 0.004$. Both differ substantially from the slope of the $3 \mathrm{D}$ best fit line, $x_{2}=0.39 \pm 0.02$. The relationship between the critical temperature and the ShultzFlory parameter was found to be highly linear for a quasi-2D geometry, but curvature was observed for the strictly 2D geometry. A chain of length $r=16$ was studied in slabs of $h$ $=1,3,5$, and 10. For $r=16$, the critical behavior remains substantially different from the bulk behavior even at large wall separations. The radius of gyration was also studied in the athermal limit, and the scaling of the 2D and 3D systems compares well with literature results. The scaling of the quasi$2 \mathrm{D}$ system trends toward that of the $2 \mathrm{D}$ system but did not reach it for the highest chain lengths studied. The $\Theta$ temperature was found by extrapolation to infinite chain length. The radius of gyration was calculated for chains at this temperature and found to conform to theoretical predictions for all geometries. For the strictly 2D geometry in particular, we find an exponent of 0.56 \pm 0.01 , close to the theoretical prediction ${ }^{14,17}$ of $R_{\mathrm{g}} \propto r^{4 / 7}$.

Acknowledgment. We acknowledge helpful discussions with Prof. Kurt Binder and Prof. Yongmei Wang, who suggested this problem to us. We also thank Thida Aye for assistance with some simulations. Funding was provided by the Department of Energy through the Computational Science Graduate Fellowship program (O.J.H.) and grant DE-FG02-01ER15121. Additional support was provided by the IRG2 group of PCCM supported by the National Science Foundation (DMR02-13706).

\section{References and Notes}

(1) Fang, F.; Szleifer, I. Langmuir 2002, 18, 5497. 802 . 
(3) Panagiotopoulos, A. Z.; Wong, V.; Floriano, M. A. Macromolecules 1998, 31, 912 .

(4) Yan, Q.; dePablo, J. J. J. Chem. Phys. 2000, 113, 5954.

(5) Cavallo, A.; Müller, M.; Binder, K. Europhys. Lett. 2003, 61, 214

(6) Shultz, A. R.; Flory, P. J. J. Am. Chem. Soc. 1952, 74, 4760.

(7) Sheng, Y.-J.; Panagiotopoulos, A. Z.; Kumar, S. K.; Szleifer, I. Macromolecules 1994, 27, 400.

(8) Mackie, A. D.; Panagiotopoulos, A. Z.; Kumar, S. K. J. Chem. Phys. 1995, 102, 1014.

(9) Dobashi, T.; Nakata, M.; Kaneko, M. J. Chem. Phys. 1980, 72, 6685.

(10) Flory, P. J. Principles of Polymer Chemistry; Cornell University Press: Ithaca, NY, 1953.

(11) Yelash, L. V.; Kraska, T.; Imre, A. R.; Rzoska, S. J. Chem. Phys. 2003, $118,6110$.

(12) Yethiraj, A. Macromolecules 2003, 36, 5854

(13) Nienhuis, B. Phys. Rev. Lett. 1982, 49, 1062.

(14) Dickman, R. J. Chem. Phys., 1992, 96, 1516.

(15) Nelson, P. H.; Hatton, T. A.; Rutledge, G. C. J. Chem. Phys. 1997, 107,1269

(16) Reiter, J.; Zifferer, G.; Olaj, O. F. Macromolecules 1989, 22, 3120

(17) Coniglio, A.; Jan, N.; Majid, I.; Stanley, H. E. Phys. Rev. B 1987, 35,3617 .
(18) Bleha, T.; Cifra, P. Polymer 2003, 44, 3745.

(19) Teraoka, I.; Wang, Y. Macromolecules 2000, 33, 6901.

(20) Rosenbluth, M. N.; Rosenbluth, A. W. J. Chem. Phys. 1955, 23 356

(21) Frenkel, D.; Smit, B. Understanding Molecular Simulation: From Algorithims to Applications, second edition; Academic Press: San Diego, CA, 2002.

(22) Escobedo, F. A.; de Pablo, J. J. J. Chem. Phys. 1996, 105, 4391.

(23) Kumar, S. K.; Szleifer, I.; Panagiotopoulos, A. Z. Phys. Rev. Lett 1991, 66, 2935

(24) Ferrenberg, A. M.; Swendsen, R. H. Phys. Rev. Lett. 1988, 61 2635.

(25) Ferrenberg, A. M.; Swendsen, R. H. Phys. Rev. Lett. 1989, 63, 1195

(26) Wilding, N. B.; Bruce, A. D. J. Phys.: Condens. Matter 1992, 4, 3087.

(27) Wilding, N. B. Phys. Rev. E 1995, 52, 602.

(28) Bruns, W. Macromolecules 1984, 17, 2826.

(29) Fisher, M. E.; Nakanishi, H. J. Chem. Phys. 1981, 75, 5857.

(30) Binder, K.; Landau, D.; Müller, M. J. Stat. Phys. 2003, 110, 1411

(31) de Gennes, P. G. Scaling Concepts in Polymer Physics; Cornell University Press: Ithaca, NY, 1979. 